\title{
Upper limits on very-high-energy gamma-ray emission from core-collapse supernovae observed with H.E.S.S.
}

H.E.S.S. Collaboration: H. Abdalla ${ }^{1}$, F. Aharonian ${ }^{3,4,5}$, F. Ait Benkhali ${ }^{3}$, E. O. Angüner ${ }^{19}$, M. Arakawa ${ }^{37}$, C. Arcaro ${ }^{1}$, C. Armand ${ }^{22}$, H. Ashkar ${ }^{17}$, M. Backes ${ }^{8,1}$, V. Barbosa Martins ${ }^{33}$, M. Barnard ${ }^{1}$, Y. Becherini ${ }^{10}$, D. Berge ${ }^{33}$, K. Bernlöhr ${ }^{3}$, R. Blackwell ${ }^{13}$, M. Böttcher ${ }^{1}$, C. Boisson ${ }^{14}$, J. Bolmont ${ }^{15}$, S. Bonnefoy ${ }^{33}$, J. Bregeon ${ }^{16}$, M. Breuhaus ${ }^{3}$, F. Brun ${ }^{17}$, P. Brun ${ }^{17}$, M. Bryan ${ }^{9}$, M. Büchele ${ }^{32}$, T. Bulik ${ }^{18}$, T. Bylund ${ }^{10}$, M. Capasso ${ }^{25}$, S. Caroff ${ }^{15}$,

A. Carosi $^{22}$, S. Casanova ${ }^{20,3}$, M. Cerruti ${ }^{15,42}$, N. Chakraborty ${ }^{3}$, T. Chand ${ }^{1}$, S. Chandra $^{1}$, R. C. G. Chaves ${ }^{16, \star \star,}$

A. Chen ${ }^{21}$, S. Colafrancesco ${ }^{21, \dagger}$, M. Curylo ${ }^{34}$, I. D. Davids ${ }^{8}$, C. Deil ${ }^{3}$, J. Devin ${ }^{24}$, P. de Wilt ${ }^{13}$, L. Dirson $^{2}$, A. Djannati-Ataï ${ }^{27}$, A. Dmytriiev ${ }^{14}$, A. Donath ${ }^{3}$, V. Doroshenko ${ }^{25}$, L. O'C. Drury ${ }^{4}$, J. Dyks ${ }^{30}$, K. Egberts ${ }^{31}$,

G. Emery ${ }^{15}$, J.-P. Ernenwein ${ }^{19}$, S. Eschbach ${ }^{32}$, K. Feijen ${ }^{13}$, S. Fegan ${ }^{26}$, A. Fiasson ${ }^{22}$, G. Fontaine ${ }^{26}$, S. Funk ${ }^{32}$, M. Füßling ${ }^{33}$, S. Gabici ${ }^{27}$, Y. A. Gallant ${ }^{16}$, F. Gaté ${ }^{22}$, G. Giavitto ${ }^{33}$, D. Glawion ${ }^{23}$, J. F. Glicenstein ${ }^{17}$,

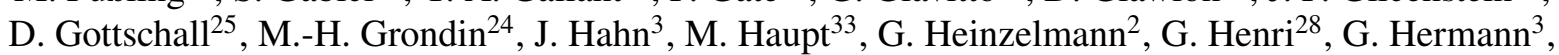

J. A. Hinton ${ }^{3}$, W. Hofmann ${ }^{3}$, C. Hoischen ${ }^{31}$, T. L. Holch ${ }^{7}$, M. Holler ${ }^{12}$, D. Horns ${ }^{2}$, D. Huber ${ }^{12}$, H. Iwasaki ${ }^{37}$, M. Jamrozy ${ }^{34}$, D. Jankowsky ${ }^{32}$, F. Jankowsky ${ }^{23}$, I. Jung-Richardt ${ }^{32}$, M. A. Kastendieck ${ }^{2}$, K. Katarzyński ${ }^{35}$, M. Katsuragawa ${ }^{38}$, U. Katz ${ }^{32}$, D. Khangulyan ${ }^{37}$, B. Khélifi ${ }^{27}$, J. King ${ }^{23}$, S. Klepser ${ }^{33}$, W. Kluźniak ${ }^{30}$, Nu. Komin ${ }^{21}$, K. Kosack ${ }^{17}$, D. Kostunin ${ }^{33}$, M. Kraus ${ }^{32}$, G. Lamanna ${ }^{22}$, J. Lau ${ }^{13}$, A. Lemière ${ }^{27}$, M. Lemoine-Goumard ${ }^{24}$, J.-P. Lenain ${ }^{15}$, E. Leser ${ }^{31,33}$, C. Levy ${ }^{15}$, T. Lohse ${ }^{7}$, R. López-Coto ${ }^{3}$, I. Lypova ${ }^{33}$, J. Mackey ${ }^{4}$, J. Majumdar ${ }^{33}$, D. Malyshev ${ }^{25}$, V. Marandon ${ }^{3}$, A. Marcowith ${ }^{16}$, A. Mares ${ }^{24}$, C. Mariaud ${ }^{26}$, G. Martí-Devesa ${ }^{12}$, R. Marx ${ }^{3}$, G. Maurin ${ }^{22}$, P. J. Meintjes ${ }^{36}$, A. M. W. Mitchell ${ }^{3,41}$, R. Moderski ${ }^{30}$, M. Mohamed ${ }^{23}$, L. Mohrmann ${ }^{32}$, J. Muller ${ }^{26}$, C. Moore ${ }^{29}$,

E. Moulin ${ }^{17}$, T. Murach ${ }^{33}$, S. Nakashima ${ }^{40}$, M. de Naurois ${ }^{26}$, H. Ndiyavala ${ }^{1}$, F. Niederwanger ${ }^{12}$, J. Niemiec ${ }^{20}$,

L. Oakes ${ }^{7}$, P. O’Brien ${ }^{29}$, H. Odaka ${ }^{39}$, S. Ohm ${ }^{33}$, E. de Ona Wilhelmi ${ }^{33}$, M. Ostrowski ${ }^{34}$, I. Oya $^{33}$, M. Panter $^{3}$,

R. D. Parsons ${ }^{3}$, C. Perennes ${ }^{15}$, P.-O. Petrucci ${ }^{28}$, B. Peyaud ${ }^{17}$, Q. Piel ${ }^{22}$, S. Pita ${ }^{27}$, V. Poireau ${ }^{22}$, A. Priyana Noel ${ }^{34}$,

D. A. Prokhorov ${ }^{21}$, H. Prokoph ${ }^{33}$, G. Pühlhofer ${ }^{25}$, M. Punch ${ }^{27,10}$, A. Quirrenbach ${ }^{23}$, S. Raab $^{32}$, R. Rauth ${ }^{12}$, A. Reimer ${ }^{12}$, O. Reimer ${ }^{12}$, Q. Remy ${ }^{16}$, M. Renaud ${ }^{16, \star}$, F. Rieger ${ }^{3}$, L. Rinchiuso ${ }^{17}$, C. Romoli ${ }^{3}$, G. Rowell ${ }^{13}$, B. Rudak ${ }^{30}$, E. Ruiz-Velasco ${ }^{3}$, V. Sahakian ${ }^{6}$, S. Saito ${ }^{37}$, D. A. Sanchez ${ }^{22}$, A. Santangelo ${ }^{25}$, M. Sasaki ${ }^{32}$, R. Schlickeiser ${ }^{11}$, F. Schüssler ${ }^{17}$, A. Schulz ${ }^{33}$, H. Schutte ${ }^{1}$, U. Schwanke ${ }^{7}$, S. Schwemmer ${ }^{23}$, M. Seglar-Arroyo ${ }^{17}$, M. Senniappan ${ }^{10}$, A. S. Seyffert ${ }^{1}$, N. Shafi ${ }^{21}$, K. Shiningayamwe ${ }^{8}$, R. Simoni ${ }^{9, \star}$, A. Sinha ${ }^{27}$, H. Sol ${ }^{14}$, A. Specovius ${ }^{32}$, M. Spir-Jacob $^{27}$,

Ł. Stawarz ${ }^{34}$, R. Steenkamp ${ }^{8}$, C. Stegmann ${ }^{31,33}$, C. Steppa ${ }^{31}$, T. Takahashi ${ }^{38}$, T. Tavernier ${ }^{17}$, A. M. Taylor ${ }^{33}$,

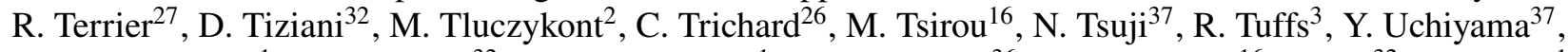
D. J. van der Walt ${ }^{1}$, C. van Eldik ${ }^{32}$, C. van Rensburg ${ }^{1}$, B. van Soelen ${ }^{36}$, G. Vasileiadis ${ }^{16}$, J. Veh $^{32}$, C. Venter $^{1}$, P. Vincent ${ }^{15}$, J. Vink ${ }^{9, \star}$, F. Voisin ${ }^{13}$, H. J. Völk ${ }^{3}$, T. Vuillaume ${ }^{22}$, Z. Wadiasingh ${ }^{1}$, S. J. Wagner ${ }^{23}$, R. White $^{3}$, A. Wierzcholska ${ }^{20,23}$, R. Yang ${ }^{3}$, H. Yoneda ${ }^{38}$, M. Zacharias ${ }^{1}$, R. Zanin ${ }^{3}$, A. A. Zdziarski ${ }^{30}$, A. Zech ${ }^{14}$, A. Ziegler ${ }^{32}$, J. Zorn ${ }^{3}$, N. Żywucka ${ }^{1}$, and N. I. Maxted ${ }^{43, \star}$

(Affiliations can be found after the references)

Received 10 February 2019 / Accepted 15 April 2019

\section{ABSTRACT}

\begin{abstract}
Young core-collapse supernovae with dense-wind progenitors may be able to accelerate cosmic-ray hadrons beyond the knee of the cosmic-ray spectrum, and this may result in measurable gamma-ray emission. We searched for gamma-ray emission from ten supernovae observed with the High Energy Stereoscopic System (H.E.S.S.) within a year of the supernova event. Nine supernovae were observed serendipitously in the H.E.S.S. data collected between December 2003 and December 2014, with exposure times ranging from 1.4 to $53 \mathrm{~h}$. In addition we observed SN 2016adj as a target of opportunity in February 2016 for $13 \mathrm{~h}$. No significant gamma-ray emission has been detected for any of the objects, and upper limits on the $>1 \mathrm{TeV}$ gamma-ray flux of the order of $\sim 10^{-13} \mathrm{~cm}^{-2} \mathrm{~s}^{-1}$ are established, corresponding to upper limits on the luminosities in the range $\sim 2 \times 10^{39}$ to $\sim 1 \times 10^{42} \mathrm{erg} \mathrm{s}^{-1}$. These values are used to place model-dependent constraints on the mass-loss rates of the progenitor stars, implying upper limits between $\sim 2 \times 10^{-5}$ and $\sim 2 \times 10^{-3} M_{\odot} \mathrm{yr}^{-1}$ under reasonable assumptions on the particle acceleration parameters.
\end{abstract}

Key words. gamma rays: general - supernovae: general - cosmic rays

${ }^{\star}$ Corresponding authors: H.E.S.S. Collaboration, e-mail: contact .hess@hess-experiment .eu

${ }^{\star \star}$ Funded by EU FP7 Marie Curie, grant agreement No. PIEF-GA-2012-332350.

$\dagger$ Deceased. 


\section{Introduction}

Despite more than a century of cosmic-ray (CR) studies, the origin of $\mathrm{CRs}^{1}$ is still not clear. The bulk of CRs detected at Earth or in space are of Galactic origin, at least for protons up to energies of $3 \times 10^{15} \mathrm{eV}$ (e.g. Ginzburg \& Syrovatskii 1964; Strong et al. 2007). Galactic CR sources can only be identified indirectly through the electromagnetic and neutrino emission caused by interactions of CRs with local gas and radiation within their sources of origin. In this respect, gamma-ray observations have proven to be an invaluable tool, as gammaray emission is uniquely associated with the presence of highly energetic CR particles. Gamma rays can be detected with sufficient statistics using gamma-ray observatories, either on-board satellites such as NASA's Fermi Large Area Telescope (LAT) in the high-energy gamma-ray domain (HE, $0.1<E<100 \mathrm{GeV}$ ), or ground-based Cherenkov telescopes, such as H.E.S.S., VERITAS, MAGIC and HAWC in the very high-energy gamma-ray domain (VHE, i.e. $E \gtrsim 50 \mathrm{GeV}$ ).

These instruments show that the prime candidate sources for Galactic CRs, supernova remnants (SNRs), are indeed gammaray sources. Young SNRs, i.e. those SNRs that are a few hundred to a few thousand years of age, emit $\mathrm{TeV}$ gamma rays at various stages during their evolution (Hewitt \& Lemoine-Goumard 2015). However, there is an ongoing debate as to whether the emission is the result of hadronic CRs interacting with ambient gas, or leptonic interactions, usually inverse Compton scattering of ambient photons by relativistic electrons. For some older remnants interacting with dense gas, the gamma-ray emission is clearly associated with hadronic CRs: the gamma-ray spectrum below $\mathrm{GeV}$ energies shows the predicted spectral feature of hadronic gamma-ray emission, usually referred to as the "pion bump" (Giuliani et al. 2011; Ackermann et al. 2013). However, there is currently no observational evidence that SNRs contain $\mathrm{CR}$ hadrons with energies around $10^{15} \mathrm{eV}(=1 \mathrm{PeV})$ or above, even for the youngest Galactic SNRs such as Cas A (Ahnen et al. 2017). This is somewhat at odds with the fact that Galactic CR hadrons need to be accelerated up to energies of at least $3 \times 10^{15} \mathrm{eV}$ to explain the knee of the CR spectrum.

The energy per supernova (SN) coupled with the Galactic $\mathrm{SN}$ rate provides enough power to sustain the CR energy density in the Galaxy (Strong et al. 2004). Moreover, it has been suggested that, for supernova remnants evolving in the winds of their progenitors, the maximum CR energy is reached in the early phase of the SNR development, within days to months after the SN event and not at the time the SNR is several hundred to thousand years old (e.g. Voelk \& Biermann 1988; Cardillo et al. 2015; Marcowith et al. 2018). The highest energy CR hadrons are then possibly accelerated in this early phase, at least for a subset of SNe (Bell et al. 2013; Zirakashvili \& Ptuskin 2016). At this early stage, less time is available for acceleration, and the shock surface area is much smaller. Only SNe exploding into sufficiently large circumstellar densities can accelerate sufficient numbers of CR proton up to energies of, or exceeding PeV energies: the number of CR hadrons being accelerated at a given time depends on the local density $n$ by the relation $\dot{N}_{\mathrm{CR}} \propto n R_{\mathrm{sh}}^{2} V_{\mathrm{sh}}$, where $R_{\mathrm{sh}}$ is the shock radius and $V_{\mathrm{sh}}$ is the shock velocity. Furthermore, the maximum energy that can be reached at given time is dependant on the magnetic field and the turbulence as shown by the

1 In the manuscript, CR refers to cosmic-ray hadrons, nuclei and electrons when not otherwise specified. following relation (e.g. Helder et al. 2012):

$$
\begin{aligned}
t & =\eta_{\mathrm{acc}} \eta_{\mathrm{g}} \frac{c E}{3 e B V_{\mathrm{sh}}^{2}} \\
& \approx 1.0 \times 10^{4}\left(\frac{\eta_{\mathrm{g}} \eta_{\mathrm{acc}}}{30}\right)\left(\frac{V_{\mathrm{sh}}}{10000 \mathrm{~km} \mathrm{~s}^{-1}}\right)^{-2}\left(\frac{B}{10 \mathrm{G}}\right)^{-1}\left(\frac{E}{100 \mathrm{TeV}}\right),
\end{aligned}
$$

with $V_{\text {sh }}$ the shock velocity, $B$ is the magnetic field strength, $t$ is the time available for acceleration in seconds, $\eta_{\mathrm{g}}$ indicates the ratio of mean free path of the particles and their gyroradius ( $\eta_{\mathrm{g}} \approx 1$, for a very turbulent magnetic field) and $\eta_{\text {acc }}=8-20$ takes into account the difference in occupancy time of particles upstream and downstream of the shock. This equation shows that in order to reach energies of $100 \mathrm{TeV}$, typically an acceleration time shorter than a day is sufficient, and for reaching the CR knee at $3 \times 10^{15} \mathrm{eV}$, an acceleration time of days to weeks is needed provided that the magnetic fields are $>1 \mathrm{G}$, instead of the $10-100 \mu \mathrm{G}$ measured in young SNRs. More detailed calculations that also take into account the expected evolution of the magnetic field strength and escape of the highest energy particles confirm these time scales, see Tatischeff (2009) and Marcowith et al. (2018).

Core-collapse (cc-)SNe originating from stellar progenitors with dense winds can fulfil the right conditions for CR acceleration, provided that the shocks are collisionless (Katz et al. 2011; Murase et al. 2011; Bell et al. 2013). First of all, the number density of a stellar wind scales as a function of radius, $n \propto \dot{M} u_{\mathrm{w}}^{-1} r^{-2}$ (where $\dot{M}$ is the mass-loss rate and $u_{\mathrm{w}}$ is the wind velocity), resulting in a circumstellar medium (CSM) density that is much higher than in the interstellar medium (ISM). As a result the cosmic-ray acceleration rate does not directly depend on the shock radius, but scales as $\dot{N}_{\mathrm{CR}} \propto \dot{M} u_{\mathrm{w}}^{-1} V_{\mathrm{sh}}$. Secondly, the magnetic fields around the shock can be amplified early on to values orders of magnitude larger than in the ISM, by the growth of CR streaming instabilities (Bell 2004). Once CR acceleration begins, it has been argued that magnetic field strengths may be amplified scaling as $B^{2} \propto n V_{\mathrm{sh}}^{2}$ (e.g. see Völk et al. 2005; Bell et al. 2013) or even $B^{2} \propto n V_{\text {sh }}^{3}$ (Vink 2012; Bell 2004), with shock velocities as fast as $20000 \mathrm{~km} \mathrm{~s}^{-1}$. SNe arising from progenitors that exhibit these high-density stellar winds are of type IIP, IIL, IIb and IIn (see the review in Chevalier \& Fransson 2017). Type IIn SNe, in particular, have been observed to eject a considerable mass prior to the explosion (Smith et al. 2008; Ofek et al. 2014; Murase et al. 2014).

Evidence for particle acceleration in cc-SNe is provided by their bright, self-absorbed synchrotron emission at radio wavelengths. These radio observations indicate the presence of relativistic electrons accelerated by strong magnetic fields, and it is thought that relativistic protons and atomic nuclei are present. One prime example is the young type IIb SN 1993J, for which a strong magnetic field, 1-100 G, and shock speeds as high as $20000 \mathrm{~km} \mathrm{~s}^{-1}$ have been estimated by Fransson \& Björnsson (1998) and Tatischeff (2009). Given these estimates and time scales for acceleration to $\mathrm{PeV}$ energies, there are good reasons to think that $\mathrm{SN}$ 1993J-like SNe can accelerate CR hadrons up to $\mathrm{PeV}$ energies within days to a few weeks (see Marcowith et al. 2018, and references therein). However, gamma-ray emission from $\mathrm{SNe}$ has not been detected so far, and upper-limits in the $\mathrm{GeV}$ domain have been established for type IIn SNe (Ackermann et al. 2015), and superluminous SN candidates (Renault-Tinacci et al. 2018).

The possibility of CR acceleration in young SNe motivates the search for signatures of $\mathrm{TeV}$ gamma-ray emission. Such 
a detection could be indicative of pion production (and subsequent decay) arising from acceleration of CR protons and nuclei beyond $\mathrm{TeV}$ energies. We analysed H.E.S.S. observations towards nine serendipitously observed cc-SN events and towards the nearby event SN 2016adj, which triggered Target of Opportunity (ToO) observations. The data selection and analysis details are presented in Sect. 2.2. Upper limits on gamma-ray flux from the direction of the CR source candidates are shown in Sect. 2.4 before constraints on the local environment of these $\mathrm{SNe}$ are derived in Sect. 3 .

\section{Observations and data analysis}

\subsection{H.E.S.S. observations}

H.E.S.S., the High Energy Stereoscopic System, is an array of five imaging atmospheric Cherenkov telescopes (IACTs) located in the Khomas Highland of Namibia at an altitude of $1800 \mathrm{~m}$ above sea level. Four 12 m-diameter telescopes (CT1-4) have been operating from December 2003 and a fifth telescope of 28 m-diameter (CT5) became operational in September 2012. In the analysis, the data of at least three telescopes (including CT5 for SN 2016adj) have been utilised. These analysis settings correspond to a field of view of $5^{\circ}$, an angular resolution $(68 \%$ containment radius) of $\sim 0.1^{\circ}$, energy threshold values spanning $\sim 200$ to $400 \mathrm{GeV}$ and an energy resolution of $\sim 15 \%$, (Aharonian et al. 2006). Nine SNe in our sample were in the field of view of other H.E.S.S.-scheduled targets, with a maximal offset of $2.5^{\circ}$ from the center of the field of view : the candidate selection is described in Sect. 2.2.1. The nearby event SN 2016adj (see Sect. 2.2.2) triggered dedicated observations a few days after the discovery date, in standard wobble mode observations (Aharonian et al. 2006) with a source offset of $\sim 0.5^{\circ}$.

\subsection{Supernova selection}

\subsubsection{Serendipitous sample}

The online $\mathrm{IAU}^{2}$ Central Bureau of Astronomical Telegrams (CBAT) supernova catalogue ${ }^{3}$ was used to compile an initial, extensive list of SN candidates. The NASA/IPAC ${ }^{4}$ Extragalactic Database (NED) ${ }^{5}$ was then queried for the redshift of each SN host galaxy to compile a short-list of SNe with redshift $z<0.01$ to ensure that only nearby $\mathrm{SNe}$ were considered. If a host galaxy was not stated for a given SN in the CBAT SN catalogue, the SN was discarded from the short-list. The H.E.S.S. database was then queried for observations in the direction of each shortlisted SN, within a time range spanning seven days prior to a year after, the $\mathrm{SN}$ discovery date. This time range was chosen to account for likely delays between the dates of the discovery and the outburst to be sure to include the peak energy suggested to occur a few days (Marcowith et al. 2014) after the SN event, but potentially lasting months. After reaching this peak emission, the gamma-ray flux is predicted to decline proportionally to $1 / t$, as we will discuss later. All H.E.S.S. data taken from December 2003 until the 31st of December 2014 were searched, and all SNe presented on CBAT on the 30th of March 2015 were considered. We removed type Ia and Ic SNe from our sample, because these types are unlikely to occur in a CSM density large enough to accelerate CRs up to TeV energies (see e.g. Smith 2014).

\footnotetext{
2 International Astronomical Union.

3 www.cbat.eps.harvard.edu/lists/Supernovae.html

Infrared Processing \& Analysis Center.

5 ned.ipac.caltech.edu/forms/z.html
}

\subsubsection{SN 2016adj}

SN 2016 adj was discovered on the 8th of February in the BOSS Survey ${ }^{6}$ (Marples et al. 2016). It appeared in the Centaurus A (Cen A, NGC 5128) galaxy, a few arcminutes from its nucleus, at a distance of $\sim 3.8 \pm 0.1 \mathrm{Mpc}$ (Harris 2010, and references therein). The SN type was soon suggested to be of type IIb by Stritzinger et al. (2016), because of the presence of a $\mathrm{H} \alpha$ line, and later confirmed by Thomas et al. (2016). The proximity and the type of the SN made it a unique and rare event for triggering VHE gamma-ray observations: the last type IIb SN occurring at a similar distance was SN 1993J, and such a nearby cc-SN could be detected at $\mathrm{TeV}$ gamma rays with current IACTs as predicted by Marcowith et al. (2014). Cen A itself was detected by H.E.S.S. after a deep observation campaign (Aharonian et al. 2009). Cen $\mathrm{A}$ is quite faint at $\mathrm{TeV}$ energies and the exposure time of a few hours considered here for SN 2016adj is too short to detect emission from the galaxy itself.

Table 1 lists the ten observed SNe along with their corresponding host galaxies, coordinates, distances (ranging between $\sim 4$ and $54 \mathrm{Mpc}$ ), types and discovery dates.

\subsection{Data analysis}

Standard quality cuts were applied to remove bad-quality data from each data set: observation runs with a minimum of three telescopes, a fraction of broken pixels $<10 \%$ and trigger-rate fluctuations $<30 \%$ were kept (see Aharonian et al. 2006). Then, for each target, the selected data were analysed using the Model analysis framework outlined in de Naurois \& Rolland (2009). For a given $\mathrm{SN}$, an $\mathrm{ON}$-region is selected centred on the $\mathrm{SN}$ position, and multiple OFF regions are selected at the same offset as the $\mathrm{ON}$ region, using the Reflected Background method (Berge et al. 2007). The $\mathrm{ON}$ region radius is $0.1^{\circ}$ for the serendipitous sample and $0.08^{\circ}$ for SN 2016adj. Results were confirmed by an independent calibration and analysis chain using the ImPACT framework (Parsons \& Hinton 2014) with standard quality cuts and the same Reflected Background method. For each 28-min observation run which passed the above-mentioned criteria, the gamma-ray excess was computed using $N_{\text {excess }}=N_{\text {on }}-\alpha N_{\text {off }}$, with $\alpha$ being the ratio of the solid angles of on and off regions. The statistical significance for each dataset was established using Eq. (17) of Li \& Ma (1983).

\subsection{Results}

No significant excess is observed for any of the SNe and flux upper limits (ULs) have been derived at the $95 \%$ confidence level under the assumption of a power law spectrum $\left(\mathrm{dN} / \mathrm{dE} \propto \mathrm{E}^{-\Gamma}\right)$ with index $\Gamma=2$. ULs have been computed using a loglikelihood approach as described in de Naurois (2012).

In Table A.1 we report the relevant statistics of the gammaray observations as described in Sect. 2.3. The total livetime, and the observational coverages expressed in days since the SN discovery date are also presented. Given the serendipitous nature of most of the observations, the livetime varies between $\sim 1$ and $\sim 50 \mathrm{~h}$, and the time delay of the first observation since the SN discovery differs between the SNe: for four objects (including SN 2016adj), H.E.S.S. observations started around, or a few days after, the discovery date, while for the other SNe, observations began much later (up to 272 days after the discovery for SN 2008 bp). The observational coverage in days after the discovery date are reported in Table A.1 and the data set for each SN

\footnotetext{
6 http://bosssupernova.com/
} 
Table 1. List of SN positions tested for H.E.S.S. gamma-ray excess emission.

\begin{tabular}{lllllll}
\hline \hline SN Name & Host galaxy & RA [J2000] & Dec [J2000] & Dist. (Mpc) & Type & Disc. date \\
\hline SN 2004cx & NGC 7755 & 23h47m52.86s & $-30^{\circ} 31^{\prime} 32.6^{\prime \prime}$ & $26 \pm 5$ & II & $2004-06-26$ \\
SN 2005dn & NGC 6861 & $20 \mathrm{~h} 11 \mathrm{~m} 11.73 \mathrm{~s}$ & $-48^{\circ} 16^{\prime} 35.5^{\prime \prime}$ & $38.4 \pm 2.7$ & II & $2005-08-27$ \\
SN 2008bk & NGC 7793 & $23 \mathrm{~h} 57 \mathrm{~m} 50.42 \mathrm{~s}$ & $-32^{\circ} 33^{\prime} 21.5^{\prime \prime}$ & $4.0 \pm 0.4$ & IIP & $2008-03-25$ \\
SN 2008bp & NGC 3095 & $10 \mathrm{~h} 00 \mathrm{~m} 01.57 \mathrm{~s}$ & $-31^{\circ} 33^{\prime} 21.8^{\prime \prime}$ & $29 \pm 6$ & IIP & $2008-04-02$ \\
SN 2008ho & NGC 922 & 02h25m04.00s & $-24^{\circ} 48^{\prime} 02.4^{\prime \prime}$ & $41.5 \pm 2.9$ & IIP & $2008-11-26$ \\
SN 2009hf & NGC 175 & 00h37m21.79s & $-19^{\circ} 56^{\prime} 42.2^{\prime \prime}$ & $53.9 \pm 3.8$ & IIP & $2009-07-09$ \\
SN 2009js & NGC 918 & 02h25m48.28s & $+18^{\circ} 29^{\prime} 25.8^{\prime \prime}$ & $16 \pm 3$ & IIP & $2009-10-11$ \\
SN 2011ja & NGC 4945 & $13 \mathrm{~h} 05 \mathrm{~m} 11.12 \mathrm{~s}$ & $-49^{\circ} 31^{\prime} 27.0^{\prime \prime}$ & $5.28 \pm 0.38$ & IIP & $2011-12-18$ \\
SN 2012cc & NGC 4419 & $12 \mathrm{~h} 26 \mathrm{~m} 56.81 \mathrm{~s}$ & $+15^{\circ} 02^{\prime} 45.5^{\prime \prime}$ & $16.5 \pm 1.1$ & II & $2012-04-29$ \\
SN 2016adj & NGC 5128 & $13 \mathrm{~h} 25 \mathrm{~m} 24.11 \mathrm{~s}$ & $-43^{\circ} 00^{\prime} 57.5^{\prime \prime}$ & $3.8 \pm 0.1$ & IIb & $2016-02-08$ \\
\hline
\end{tabular}

Notes. The list was compiled using a system of cuts described in Sect. 2.2. The name, host galaxy, coordinates, estimated distance, SN type and discovery date are given for each SN.

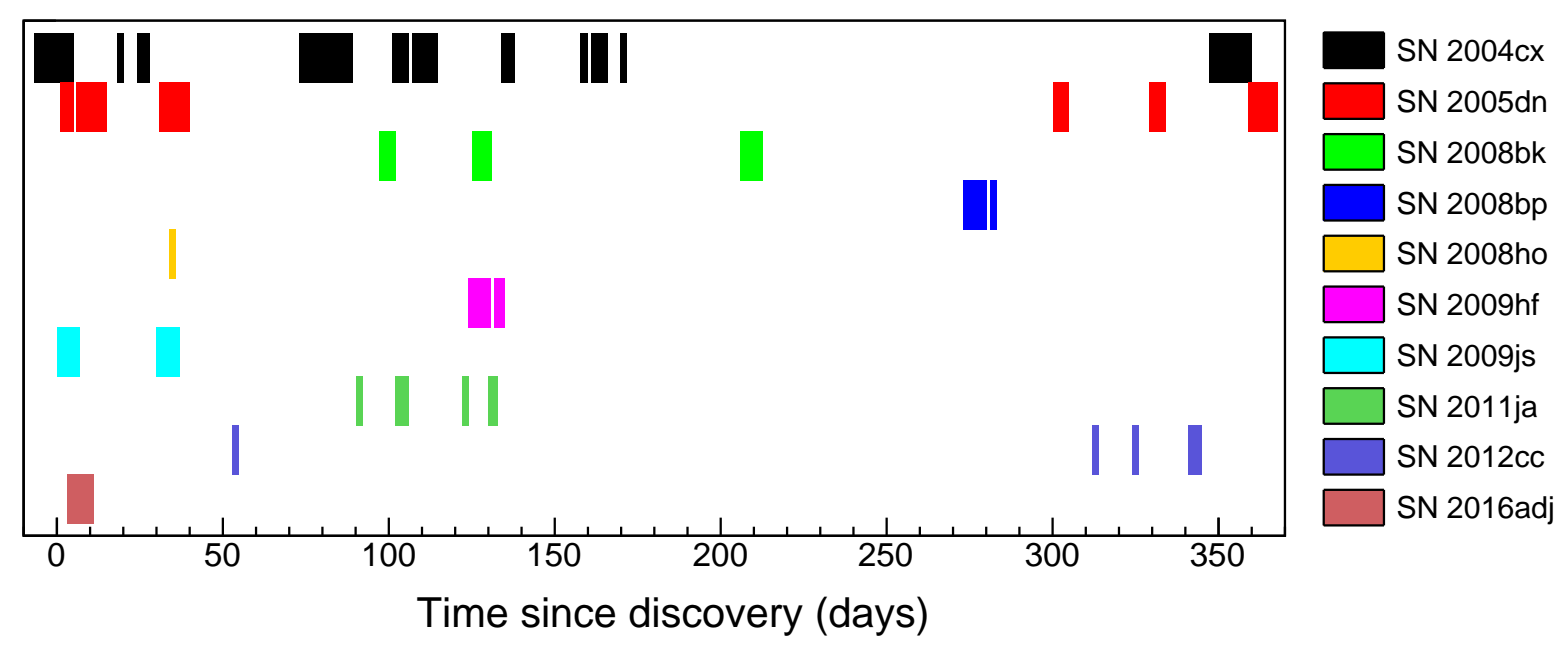

Fig. 1. H.E.S.S. observation windows for each SN selected for analysis. Time $=0$ corresponds to the SN discovery date reported in literature.

is represented in Fig. 1.The average time delays, weighted by the exposure of individual observation periods (as represented on Fig. 1), are also reported in Table A.1. SN 2016adj was observed every night from day 3 till day 10 after the discovery date, and the average time delay amounts to $\sim 7$ days.

In Table 2, ULs on the integrated flux above the energy threshold and above $1 \mathrm{TeV}$ are presented. The value of $1 \mathrm{TeV}$ corresponds to the optimal H.E.S.S. sensitivity: it is chosen to compare all results, as the energy threshold depends on observational conditions and varies as indicated in the table. For four SNe (2004cx, 2008bk, 2008bp, 2009js) these ULs supersede previous preliminary results (Lennarz \& the H.E.S.S. Collaboration 2013), confirming the non-detections with better sensitivity. In column five of Table 2, ULs on the luminosities are presented for each object: these ULs are computed above the energy threshold and above $1 \mathrm{TeV}$, using the distance to the host galaxy (see Table 1). Errors on the distances are not taken into account. The luminosity values above the energy threshold span a range from $\sim 2 \times 10^{39}$ to $\sim 1 \times 10^{42} \mathrm{erg} \mathrm{s}^{-1}$. This range is mainly due to the differences in the source distances and to the offset angle with respect to the observation position, observations with large offcenter angles having a reduced detection sensitivity. Note that these ULs correspond to a gamma-ray fluence within a year of $\sim 6 \times 10^{46}$ to $\sim 3 \times 10^{49} \mathrm{erg}$, corresponding to $0.006-3 \%$ of the canonical SN explosion energy of $10^{51} \mathrm{erg}$.
We carefully checked that no significant gamma-ray peak occurred during the duration of the observations. As an example, Fig. 2 shows the time evolution of the flux above $1 \mathrm{TeV}$ for SN 2016adj, consistent with zero during the observing period. This is the case for all the other SNe (see Appendix B.1). The lightcurves presented are binned on a nightly basis and we also checked that no significant emission occurred on a weekly basis for any of the objects. Error bars correspond to $68 \%$ confidence levels. This confirms that no significant $\mathrm{TeV}$ emission is found towards any of the $\mathrm{SNe}$ within one year of the initial explosion.

\section{Discussion}

The serendipitous nature of the observations provided us with a sample with a large diversity in distances, post-explosion delay times and observing conditions. This has to be kept in mind when interpreting the non-detection of $\mathrm{TeV}$ emission from these SNe. For instance, two nearby SNe (SN 2008bk, SN 2011ja) have been observed around 100 days after the discovery and we might have missed the periods of maximum $\mathrm{TeV}$ emission. By contrast, early and relatively deep observations towards SN $2004 \mathrm{cx}$ and SN 2005dn have been performed, but these two SNe are beyond $20 \mathrm{Mpc}$ in distance, and this may explain their nondetection. For SN 2016adj, as already mentioned, the candidate 
Table 2. Upper limits (ULs) on the integrated flux above the energy threshold and above $1 \mathrm{TeV}$.

\begin{tabular}{lccccccc}
\hline \hline SNe & $E_{\mathrm{Th}}$ & $\begin{array}{c}\text { Flux UL } \\
\left(>E_{\mathrm{Th}}\right) \\
\left(10^{-13} \mathrm{~cm}^{-2} \mathrm{~s}^{-1}\right)\end{array}$ & $\begin{array}{c}\text { Flux UL } \\
(>1 \mathrm{TeV})\end{array}$ & $\begin{array}{c}\text { UL on } L \\
\left(>E_{\mathrm{Th}}\right) \\
\left(10^{40} \mathrm{erg} \mathrm{s}^{-1}\right)\end{array}$ & $\begin{array}{c}\text { UL on } L \\
(>1 \mathrm{TeV})\end{array}$ & $\begin{array}{c}\text { ULs on } \dot{M} / u_{\mathrm{w}} \\
\left(10^{-5} M_{\odot} \mathrm{yr}^{-1} \mathrm{~km}^{-1} \mathrm{~s}\right)\end{array}$ \\
\hline SN 2004cx & 0.18 & 10 & 1.9 & 13.0 & 2.5 & 6.7 & 3.2 \\
SN 2005dn & 0.21 & 2.2 & 0.41 & 6.2 & 1.2 & 3.8 & 0.26 \\
SN 2008bk & 0.21 & 6.0 & 4.8 & 0.18 & 0.15 & 1.4 & 0.4 \\
SN 2008bp & 0.21 & 29 & 5.5 & 46.7 & 8.9 & 15.9 & 12.3 \\
SN 2008ho & 0.33 & 16 & 7.7 & 52.8 & 25.4 & 9.4 & 5.3 \\
SN 2009hf & 0.21 & 20 & 5.3 & 111 & 29.5 & 19.9 & 15.9 \\
SN 2009js & 0.63 & 15 & 11 & 7.3 & 5.4 & 3.1 & 0.9 \\
SN 2011ja & 0.21 & 20 & 5.2 & 1.1 & 0.28 & 1.77 & 1.6 \\
SN 2012cc & 0.72 & 15 & 10 & 11.5 & 7.7 & 11.6 & 3.7 \\
SN 2016adj & 0.196 & 8.8 & 1.7 & 0.24 & 0.05 & 0.25 & 0.20 \\
\hline
\end{tabular}

Notes. These ULs are computed assuming 95\% confidence level and a power-law index of 2 . The associated ULs on the luminosities are computed using the distances reported in Table 1. Upper limits on $\dot{M} / u_{\mathrm{w}}$ are derived from methods using the average time and a fit on the LCs, both using Eq. (3) (see text).

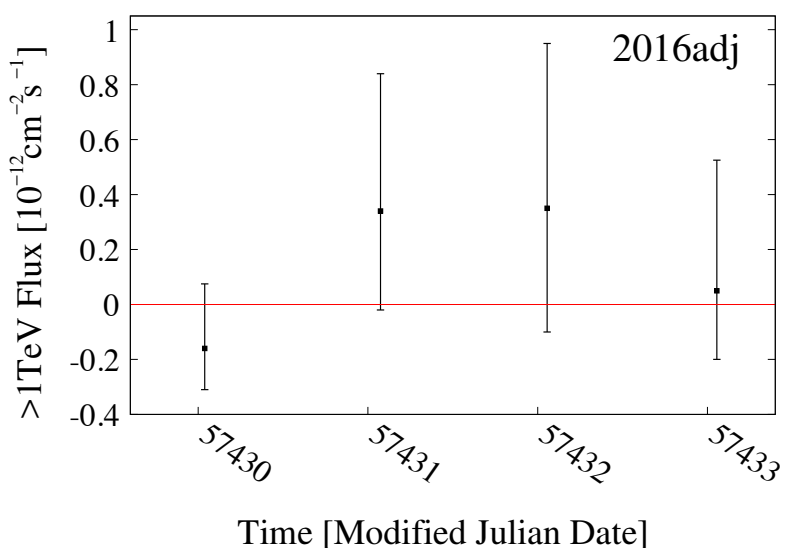

Fig. 2. Light curve of SN 2016adj. Data points are binned on a nightly basis, the red solid line indicates the zero level.

was very promising in terms of distance and time delay, although the duration of observations was not as long as initially planned due to bad weather conditions. Despite the diversity in the observed sample, these H.E.S.S. observations can be used to derive constraints on a key parameter impacting the SN gammaray luminosity, namely the mass-loss rate of the progenitor star, which determines the CSM density.

The sample consists mostly of type IIP SNe, the most commonly observed type of cc-SNe, for which the progenitor is a cool red supergiant (RSG) star, like the progenitor found for SN 2003gd (Smartt et al. 2004). Several type IIP SNe show evidence for interaction with dense environments in the form of non-thermal radio emission and X-ray emission (e.g. Chevalier 1982a; Pooley et al. 2002). These progenitors are believed to have mass-loss rates of typically $10^{-6}-10^{-4} M_{\odot} \mathrm{yr}^{-1}$. However, some progenitors appear to exhibit mass-loss rates as high as $10^{-4}$ $10^{-3} M_{\odot} \mathrm{yr}^{-1}$ (Smith 2014). When combined with the relatively slow RSG winds (Chevalier \& Fransson 2017), these mass-loss rates may lead to the right circumstances in terms of density for particle acceleration to proceed up to very high energies and for gamma-ray emission to be potentially detectable (e.g. Moriya et al. 2011; Marcowith et al. 2014). Moreover, there is accumulating evidence of enhanced mass-loss rates from progenitors in the last years prior to explosion (e.g. Fuller 2017), which may similarly lead to enhanced $\mathrm{CR}$ proton acceleration and gamma-ray emission.

For type IIb SNe, like SN 2016adj, the mass-loss rate is predicted to be typically over $10^{-5} M_{\odot} \mathrm{yr}^{-1}$ for a wind velocity of $u_{\mathrm{w}} \simeq 10 \mathrm{~km} \mathrm{~s}^{-1}$, as estimated for SN 1993J (Tatischeff 2009). However, there is evidence for a sub-type of type IIb SNe depending on the compactness of the progenitor. A more compact, less luminous progenitor with a lower $\mathrm{H}$ mass envelope and a high-speed wind, would produce a lower density environment (Chevalier \& Soderberg 2010). We will refer to this type of SNe as compact type IIb. Below we will discuss the implications of the obtained H.E.S.S. ULs in terms of the mass-loss rates of the ten observed SNe.

\subsection{Modelling}

To place our flux upper limits into the context of the SN environment, we use a semi-analytical model for cc-SNe described in Dwarkadas (2013). The author predicts a gamma-ray flux of hadronic origin from $\mathrm{SNe}$ and young SNRs based on the hydrodynamical evolution described in Chevalier (1982b) and Chevalier \& Fransson (1994), and the gamma-ray emissivity formula prescribed by Drury et al. (1994). The model assumes a constant stellar mass-loss rate and wind velocity, usually known as the steady wind scenario (Chevalier 1982b). Under this assumption, the CSM density is given by the continuity of mass equation: $\rho_{\mathrm{amb}}=\dot{M} / 4 \pi u_{\mathrm{w}} r^{-2}$, which shows that a combination of a high mass-loss rate and a low wind velocity will lead to a high-density CSM. Low wind speeds of $\sim 10 \mathrm{~km} \mathrm{~s}^{-1}$ are commonly realised in RSG progenitors (e.g. Smith et al. 2009).

For cc-SNe, the model of Dwarkadas (2013) gives the following relation of the expected gamma-ray flux as a function of stellar mass-loss parameters, SN explosion characteristics and time, $t$, since the explosion:

$F_{\gamma}\left(>E_{0}, t\right)=\frac{3 q_{\alpha} \xi\left(\kappa C_{1}\right) m^{3}}{32 \pi^{2}(3 m-2) \beta \mu m_{\mathrm{p}} d^{2}}\left[\frac{\dot{M}}{u_{\mathrm{w}}}\right]^{2} t^{m-2}$.

This equation is valid as long as the maximum photon energy $E_{\mathrm{ph} \text {,max }}$ (related to the maximum energy of accelerated 
particles $\left.E_{\max }\right)$ is significantly higher than $E_{0}$. As shown by Marcowith et al. (2018), in the case of cc SNe evolving in their dense wind progenitor such as SN 1993J, $E_{\max }$ conservatively remains above $\sim 0.5 \mathrm{PeV}$ (i.e. $E_{\mathrm{ph}, \max } \gtrsim 30 \mathrm{TeV}$ ) during the first year after the $\mathrm{SN}$, so that Eq. (1) can be used as is. The variable $t$ is the elapsed time in days since the SN explosion, and the variable $d$ is the distance in Mpc as given in Table 1. The variable $q_{\alpha}$ is the gamma-ray emissivity normalised to the hadronic CR energy density, for which values are tabulated in Drury et al. (1994). We use $q_{\alpha}(\geq 1 \mathrm{TeV})=1.02 \times 10^{-17} \mathrm{~s}^{-1} \mathrm{erg}^{-1} \mathrm{~cm}^{3}$

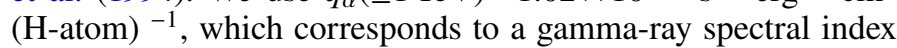
of 2, adopting the value assumed for SN 1993J in Tatischeff (2009). This study clearly shows that the sub shock and total compression ratios both are close to 4 , meaning the shock remains weakly modified throughout the SN 1993J time evolution. The potential systematic error introduced by this assumption can be quantified by considering the extreme case of a steep spectral index of 2.4. In such a case, the gamma-ray emissivity would become $q_{\alpha}(\geq 1 \mathrm{TeV})=8.1 \times 10^{-19} \mathrm{~s}^{-1} \mathrm{erg}^{-1} \mathrm{~cm}^{3}$

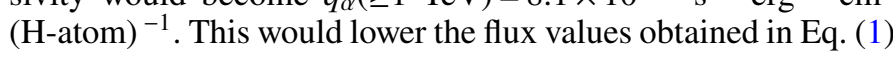
by a factor of $\sim 12$. The parameter $\xi$ is the fraction of the shock energy flux that is converted into CR proton energy, and $\beta$ is the fraction of the total volume, $\mathcal{V}$, that is already shocked and where the density of target protons is high $\left(\mathcal{V}_{\text {shocked }}=\beta 4 \pi R_{\mathrm{sh}}^{3} / 3\right)$. $m_{\mathrm{p}}$ is the proton mass, and $\mu$ the mean molecular weight of the nuclear targets in the CSM. We set $\xi$ equal to $0.1, \beta$ to 0.5 and $\mu=1.4$ following the parameters chosen for cc-SNe in Dwarkadas (2013). Finally, the parameter $\kappa$ is the ratio of the forward shock (FS) radius to the contact discontinuity (CD) radius. $C_{1}$ is a constant that can be expressed in terms of the geometry of the explosion, as the radius of the FS is defined as $R_{\mathrm{sh}}=\kappa R_{\mathrm{CD}}=\kappa C_{1} t^{m}$.

For this study, we substitute $\kappa C_{1}$ with $V_{\text {sh }} /\left(m t^{m-1}\right)$ where $V_{\text {sh }}$ is the shock velocity and $m$ is the expansion parameter, leading to the following relation:

$F_{\gamma}\left(E_{0}, t\right)=\frac{3 q_{\alpha} \xi\left(V_{\mathrm{sh}}\right) m^{2}}{32 \pi^{2}(3 m-2) \beta \mu m_{\mathrm{p}}}\left[\frac{\dot{M}}{u_{\mathrm{w}}}\right]^{2}\left(\frac{1}{d^{2}}\right)\left(\frac{1}{t}\right)$.

According to the model of Chevalier (1982a) for a steady wind scenario, $m$ can be expressed as $m=(n-3) /(n-2)$, where $n$ is the index of the ejecta density profile $\left(\rho_{\mathrm{ej}} \propto r^{-n}\right)$. For $n$, Chevalier (1982a) has found values between 7 and 12, implying that $m$ lies between 0.8 and 0.9 , in agreement with observations of some radio SNe (e.g. Weiler 2006). We adopt here $m=0.85$, and a shock velocity $V_{\mathrm{sh}}=10000 \mathrm{~km} \mathrm{~s}^{-1}$ as fiducial parameters. The dependence on $1 / t$ breaks down for $t \rightarrow 0$, but particle acceleration does not immediately start at $t=0$, as the shock first needs to break out of the star, and some time (days-weeks, depending on the B-field value and the turbulence) should be allowed for the particles to be accelerated to high enough energies to produce VHE gamma rays. Note also that $t=0$ should refer to the time of core collapse, whereas in our analysis we had to use the time of SN detection, which could be several hours or even days after the actual explosion time. Note that within the first week after core collapse the SN may be so bright in the optical band that the gamma-ray emission is strongly attenuated by gamma-gamma interactions, as explained in Sect. 3.3.

Our ULs on the gamma-ray flux above $1 \mathrm{TeV}$ given in Table 2 can be converted into an upper value for $\dot{M} / u_{\mathrm{w}}$ by inverting Eq. (2), replacing the constant parameters by their respective values and expressing the mass-loss rate such that

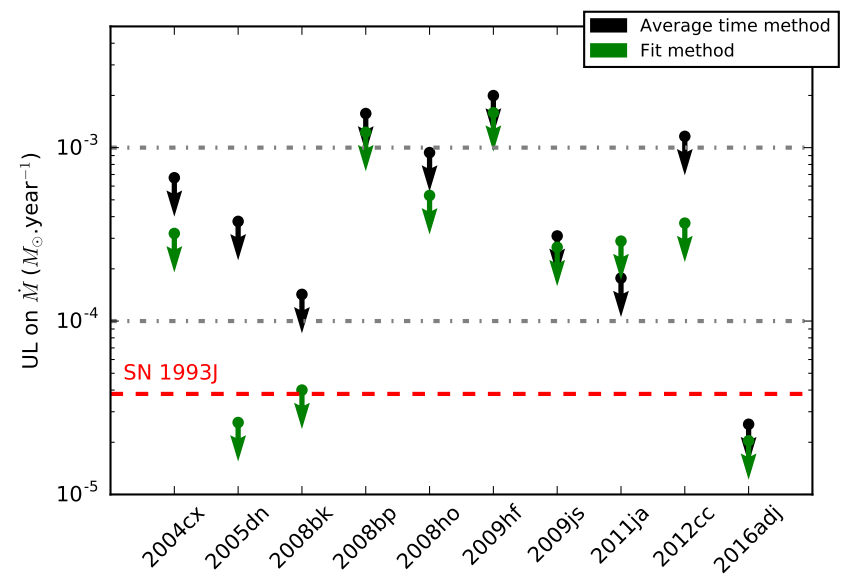

Fig. 3. Upper limits on progenitor mass-loss rates, $\dot{M}$, assuming $u_{\mathrm{w}}=$ $10 \mathrm{~km} \mathrm{~s}^{-1}$ for the ten cc-SNe investigated in this study, derived with two methods (see text). The mass-loss rate for SN 1993J, derived by Tatischeff (2009), is also shown.

$\dot{M}=10^{-5} \quad \dot{M}_{-5} M_{\odot} \quad \mathrm{yr}^{-1} \quad$ and the wind velocity

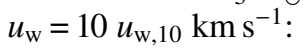

$\left[\frac{\dot{M}_{-5}}{u_{\mathrm{w}, 10}}\right]^{2} \leq \frac{F_{\gamma}(>1 \mathrm{TeV}) d_{\mathrm{Mpc}}^{2} t_{\mathrm{day}}}{5.14 \times 10^{-12}}$.

The numerical value of $5.14 \times 10^{-12}$ is of the same order as that derived in Tatischeff (2009) for SN 1993J (within a factor $\lesssim 2$ ), and comparable to that obtained by Murase et al. (2014) with the same parameters, within a factor $\lesssim 4$.

To establish the ULs on $\dot{M} / u_{\mathrm{w}}$, we use two methods: the first one simply consists in substituting the variable $t$ by the exposureweighted average time reported in Table A.1. A second method consists in fitting with Eq. (3) the nightly binned flux points (cf. Figs. 2 and B.1) with their respective dates expressed relatively to the discovery date. For this method, we set the fitting function given in Eq. (3) to zero at $t<5$ days, in order to account for the possibly strong gamma-ray attenuation through gammagamma interactions during the early stages of the SN evolution (see Sect. 3.3). The goodness of the fit is estimated by a $\chi^{2}$ test. Fitting the lightcurves in order to constrain the mass-loss parameter is very sensitive to the gamma-ray flux immediately following the SN explosion, whereas using the exposure-weighted time $t$ is more sensitive to the average measured flux. Results and methods are discussed in the next section.

\subsection{Derived upper limits on the wind properties}

Upper limits representing a $2 \sigma$ level of the $\dot{M} / u_{\mathrm{w}}$ ratio, derived from the two methods described in the previous section, are given in Table 2 in units of $10^{-5} M_{\odot} \mathrm{yr}^{-1} \mathrm{~km}^{-1} \mathrm{~s}$. The constraints on $\dot{M}$, assuming $u_{\mathrm{w}}=10 \mathrm{~km} \mathrm{~s}^{-1}$, are shown in Fig. 3 .

In general, more constraining ULs are obtained through the fit to the lightcurves, as compared to the method using the timeaverage flux limits: this is the case for SN 2004cx, and SN $2005 \mathrm{dn}$, for which fluxes are determined shortly after the SN explosion dates, as well as as observation spanning the whole year. For $2005 \mathrm{dn}$, the value obtained with the fit method seem to favor the first sets of flux points mostly negatives with small errors, compared to late observations taken after a gap which are showing more positive values. The same case seems to happen for SN 2008bk, for which observations were all taken $\sim 100$ days 
after the discovery but are spanning over several months. For SNe observed only during a short time span, like SN 2009hf, SN 2011ja and SN 2016adj, the two methods give similar ULs, as expected. The method using a fit to Eq. (3) gives more weight to the early observations, as this is where the highest fluxes are expected and relies on the assumption that the gamma-ray flux evolution follows exactly the $1 / t$ scaling. In reality, the progenitor mass loss history may be more complicated, and there is also some uncertainty regarding the onset of particle acceleration and the effect of gamma-gamma attenuation. As such, the time-average flux method gives perhaps a less precise but more conservative constraints on $\dot{M} / u_{\mathrm{w}}$.

For the nine $\mathrm{SNe}$ of the serendipitous sample, both methods give ULs lying between $\sim 2.0 \times 10^{-5}$ and $\sim 2.0 \times 10^{-3} M_{\odot} \mathrm{yr}^{-1}$, and are consistent with predictions for type IIP $\mathrm{SNe}$ with a RSG progenitor having a mass-loss rate in the range $10^{-6}$ $10^{-4} M_{\odot} \mathrm{yr}^{-1}$. These constraints show large values in $\dot{M} / u_{\mathrm{w}}$, but still within the range of expected mass-loss rates for some RSGs.

For SN 2016adj, the mass-loss rate UL, confirmed by both methods, is reaching $\sim 2.5 \times 10^{-5} M_{\odot} \mathrm{yr}^{-1}$, as the SN occurred in the nearby Cen A galaxy and has been observed very early: it is of the same order as the value estimated for the well-studied SN 1993J $\left(\dot{M}=3.5 \times 10^{-5} M_{\odot} \mathrm{yr}^{-1}\right.$ for $u_{w}=10 \mathrm{~km} \mathrm{~s}^{-1}$, Tatischeff 2009), both SNe being of Type IIb occurring at similar distances. We note that the expansion parameter of SN 1993J near the time of discovery is estimated to be high, $m=0.919 \pm 0.09$, as implied by early radio observations (Bartel et al. 2002). Apart from this difference, given that the TeV gamma-ray flux from SN 1993J was predicted to be at the level of sensitivity of current IACTs (Marcowith et al. 2014), TeV emission from SN 2016adj could have been detected with H.E.S.S. if it were to share the same environmental properties as SN 1993J.

\subsection{Opacity due to gamma-gamma absorption.}

At short timescales after the explosion, when the $\mathrm{SN}$ is near maximum optical peak luminosity, the VHE gamma-ray emission may be suppressed due to electron-positron pair-production, arising from the interaction of $\mathrm{TeV}$ photons with low-energy photons from the SN photosphere. This gamma-gamma absorption critically depends on the photosphere properties of each object, and no complete model exists so far. First attempt to quantify this effect in SN 1993J has been carried out by Tatischeff (2009) under the assumption of isotropic interactions with photospheric photons. More recently, preliminary calculations of this time-dependent absorption accounting for the geometrical effects have been performed by Marcowith et al. (2014) in the case of SN 1993J for which the parameters of the SN hydrodynamical evolution and photosphere are well known. While the absorption is expected to be very large during the first week, the attenuation amounts to a factor of a few at $t \sim 10$ 20 days before gradually decreasing at the level of $\sim 10-20 \%$ on a month timescale. Thus, pair-production may have an impact on the detectability of gamma-ray emission from the $\mathrm{SNe}$ in the first week after the explosion, depending on the luminosity evolution of a given SN. Given the large spread in the distribution of time delays between the H.E.S.S. observations and the SN discoveries for the SN serendipitous sample, this opacity can, in general, be ignored.

The only object for which this attenuation should be considered is SN 2016adj, whose ToO observations are clustered at short time delays. If the attenuation of $\mathrm{TeV}$ photons is at a similar level as that estimated in SN 1993J, the expected VHE gamma-ray flux would be much lower during a large part of the H.E.S.S. observation time window and this would explain the non-detection of any excess towards SN 2016adj. In other words, constraints on the VHE gamma-ray flux could not be directly translated into limits on the mass-loss rate until the above-mentioned parameters regarding the evolution of the $\mathrm{SN}$ shock and photosphere are better known. SN 2016adj occurred very close to the center of Cen A, so it may well be that the local environment led to an additional gamma-gamma absorption and this could also explain the non-detection.

\subsection{Discussion on the SNe environment in perspective of other observations.}

There may be another explanation for the non-detection of SN 2016adj, for which the ULs are near or below the predicted gamma-ray flux, namely that this interesting candidate does not present the right environmental properties. This could be the case if SN 2016adj was a compact type IIb SN (cIIb), as opposed to SN 1993J, which is known to have been an extended type IIb (eIIb, Chevalier \& Soderberg 2010). For type cIIb SNe, the wind velocity is probably higher $\left(u_{\mathrm{w}} \gtrsim 100 \mathrm{~km} \mathrm{~s}^{-1}\right)$, implying a lower density CSM. Under the hypothesis that SN 2016adj is of type cIIb, assuming $u_{\mathrm{w}}=100 \mathrm{~km} \mathrm{~s}^{-1}$, the upper limit on the mass-loss rate would increase by a factor of 10 (see Eq. (3)), i.e. $\dot{M}<3.0 \times 10^{-4} M_{\odot} \mathrm{yr}^{-1}$, and could better accommodate the H.E.S.S. non-detection of this apparent SN 1993J twin. Van Dyk et al. (2016) proposed that SN 2016adj originated from a progenitor with a lower luminosity than the SN 1993J progenitor, suggesting that the explosion might be of compact IIb-type. This claim, however, was later disfavoured by Sugerman \& Lawrence (2016). Further multi-wavelength observations will weigh in on the nature of this object, and an analysis of radio and X-ray data is forthcoming (Hajela et al., in prep.). For the rest of the sample, in addition of being Type II SNe (mostly IIP), the nine selected candidates did not have any strong indication of the conditions necessary for significant early CR acceleration. Several of the $\mathrm{SNe}$ have been studied at various levels of detail, with results consistent with our study. Radio and X-ray observations are of prime interest to check the consistency of our result, as they can provide additional constraints on the wind parameters. The literature reports radio and X-ray observations for only one SNe in our sample, namely for the rather close-by object SN 2011ja. Chakraborti et al. (2013) suggest that the measured SN 2011ja radio and $\mathrm{X}$-ray fluxes are consistent with an expansion into a low-density bubble and interaction with an inhomogeneous circumstellar medium formed by a RSG $\left(\sim 12 M_{\odot}\right)$. The corresponding progenitor mass-loss rate was estimated to be of the order of $\sim 10^{-6} M_{\odot} \mathrm{yr}^{-1}$, consistent with the constraint of $<1.6 \times 10^{-4} M_{\odot} \mathrm{yr}^{-1}$ derived from our non-detection at $\mathrm{TeV}$ gamma rays.

SN 2008bk was observed to have a progenitor mass of $\sim 8 M_{\odot}$ and a luminosity of $\log \left(L / L_{\odot}\right) \sim 4.5$ (Davies \& Beasor 2018) after its post-explosion disappearance was confirmed by Mattila et al. (2013) and Van Dyk (2013). By applying the de Jager et al. (1988) prescription (see also Mauron \& Josselin 2011), the observed luminosity implies a pre-SN progenitor mass-loss rate of $\sim 10^{-6.3} M_{\odot} \mathrm{yr}^{-1}$, consistent with our model-dependent constraint of $<1.4 \times 10^{-4} M_{\odot} \mathrm{yr}^{-1}$.

We further mention a study of the spectral evolution of 122 nearby $\mathrm{SNe}$ including post-explosion spectral line observations of SN 2008bp at wavelengths 4000-9500 $\AA$ at 12, 40 and 47 days (Gutiérrez et al. 2017). The authors noted SN 2008bp to be the only event to not have $\mathrm{Fe}$-group and $\mathrm{H}_{\gamma}$ line-blending in early stages of evolution. This characteristic might suggest that the 


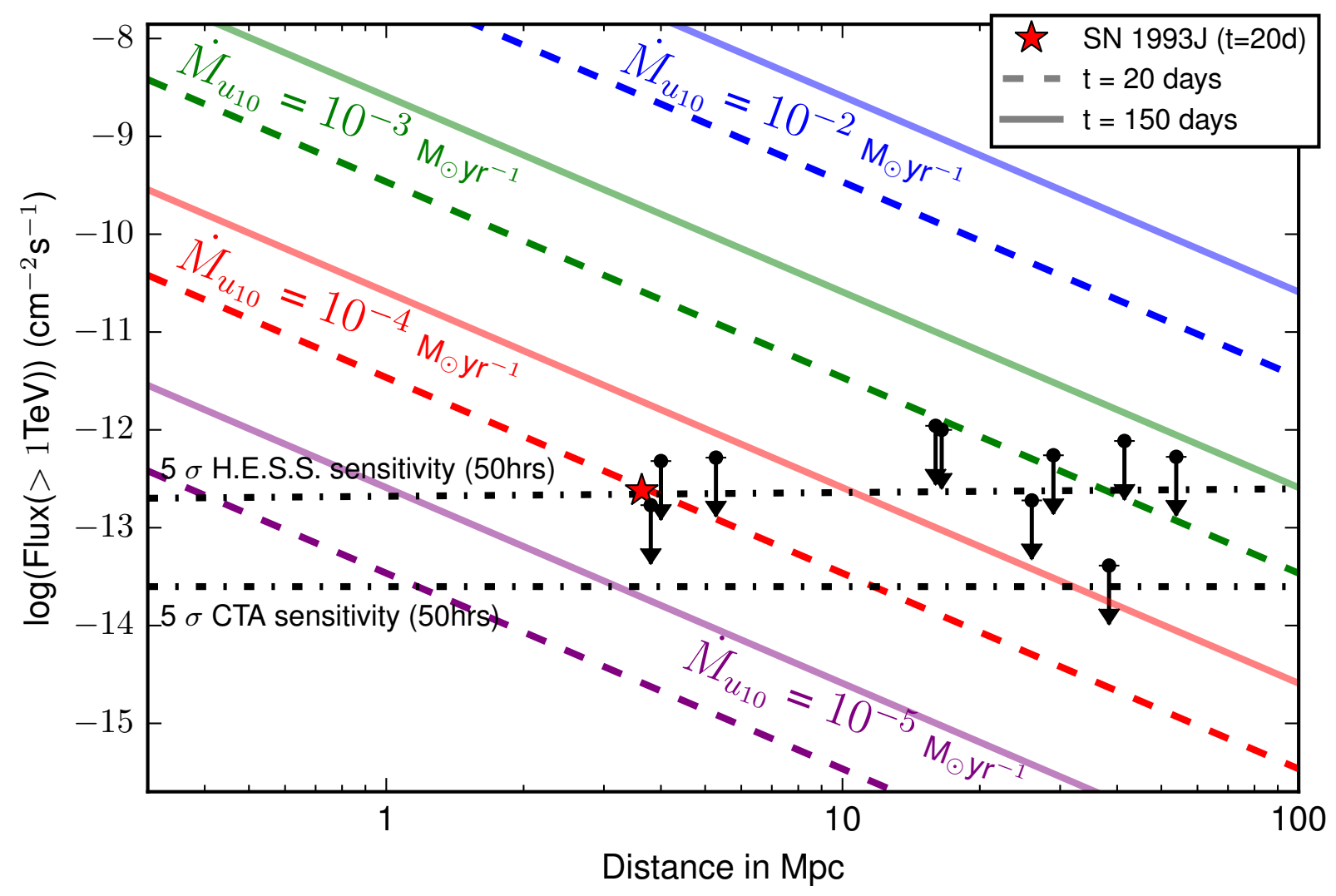

Fig. 4. Predicted flux above $1 \mathrm{TeV}$ using Eq. (1) as a function of the distance to the source. Mass-loss rates are given in units of $M_{\odot} \mathrm{yr}^{-1}$ assuming $u_{\mathrm{w}}=10 \mathrm{~km} \mathrm{~s}^{-1}$ and the parameters described in Sect. 3.1, for $t=20$ days (solid lines) and $t=150$ days (dashed lines) after the SN explosion. The Mass-loss rate is given for each pair of lines of the same color. The expected flux for SN 1993J is computed using Eq. (1) and $t=20$ days. The CTA sensitivity for a $50 \mathrm{~h}$ long observation is taken from Acharya et al. (2013). The ten ULs on the flux above 1 TeV derived in this study are also shown (see text).

circumstellar medium of SN 2008bp was less dense than that of the rest of the sample. Outlier behaviour was also discovered for SN 2009js. Gandhi et al. (2013) found the event to be subluminous, suggesting a low ejecta mass and explosion energy. It follows that the environments of SN 2008bp and SN 2009js are not likely conducive to the $\mathrm{TeV}$ gamma-ray detection sought in our study, but are consistent with the mass-loss rate upper-limits of $\sim 1.6 \times 10^{-3}$ and $\sim 3.1 \times 10^{-4} M_{\odot} \mathrm{yr}^{-1}$, respectively, as derived from the H.E.S.S. observations.

\subsection{Prospects for future observations}

In order to put the flux ULs derived in this work in perspective, Fig. 4 shows the gamma-ray flux computed with Eq. (2) as a function of the distance for different values of the pre-SN mass-loss rate, together with the typical five sigma point-source sensitivities of H.E.S.S. and of the next generation of IACTs, the Cherenkov Telescope Array (CTA, Acharya et al. 2013). For comparison our upper limits are shown, which, as expected, are close to the $50 \mathrm{~h}$ H.E.S.S. sensitivity, $\simeq 2 \times 10^{-13} \mathrm{~cm}^{-2} \mathrm{~s}^{-1}$ (Aharonian et al. 2006), bearing in mind the exposure times. Parameters are chosen as described in Sect. 3.1, with $q_{\alpha}$ corresponding to a flat spectral index. Two different values for the time delay since the SN explosion are considered: $t=150$ days corresponds to a value that is representative of our sample, whereas $t=20$ days roughly corresponds to the optical peak luminosity of a SN. As seen in Fig. 4, the VHE gamma-ray flux at $t=20$ days from a SN 1993J-like event is within reach with current IACTs like H.E.S.S., and would clearly be detected by CTA. At $t=150$ days, and for mass-loss rates higher than $10^{-4} M_{\odot} \mathrm{yr}^{-1}$, CTA may detect cc-SNe as far out as the Virgo cluster (16 Mpc).

Mass-loss rates above $10^{-4} M_{\odot} \mathrm{yr}^{-1}$ are not uncommon, but are usually confined to Type IIb and IIn SNe. Each of these types represent, respectively, $\sim 10$ and $\sim 8 \%$ of the total cc-SN rates according to Smith et al. (2011). The number of cc-SNe occurring in a year can roughly be expressed as a function of the total available stellar mass in $10^{10} M_{\odot}$ units. In our Galaxy, this implies a cc-SN rate of about two per century (see e.g. Li et al. 2011). A galaxy cluster in the local Universe, as the Virgo cluster, has a total stellar mass of the order of $10^{13} M_{\odot}$ (see e.g. O'Sullivan et al. 2017), which would bring the number of cc-SNe up to $\sim 10$ per year in a near radius of $16 \mathrm{Mpc}$. This number is very similar to the number of objects predicted by Horiuchi et al. (2011) for a volume of radius $\lesssim 10 \mathrm{Mpc}$. Another example is given by Smartt et al. (2009), who identified 5 type IIP SNe in 1999 within $18 \mathrm{Mpc}$, which may represent 60-70\% (see e.g. Smartt et al. 2009; Smith et al. 2011) of the whole sample of cc-SNe of that year. Note that type II objects can also exhibit enhanced pre-SNe mass-loss rates above $10^{-3} M_{\odot} \mathrm{yr}^{-1}$ (e.g. Das \& Ray 2017; Arcavi et al. 2017), and other studies have shown that such high mass-loss rates are not so rare among type IIb SNe (Fuller 2017; Ouchi \& Maeda 2017). It is then reasonable to expect $\sim 1$ to 2 cc-SNe with $\dot{M}>10^{-4} M_{\odot} \mathrm{yr}^{-1}$ occurring per year, within a radius of $16 \mathrm{Mpc}$, whatever the sub-type. Such nearby cc-SN events offer a great opportunity for the detection of gamma rays using IACT observations triggered by observations at optical wavelengths. Such a ToO program 
to observe cc-SNe as distant as $10 \mathrm{Mpc}$ is currently in place within the H.E.S.S. collaboration. For the expected gamma-ray luminosities of supernovae, the wide-field $\mathrm{TeV}$ observatories as HAWC (Abeysekara et al. 2013) and in the future LHAASO Vernetto \& LHAASO (2016) are less ideally positioned for detecting gamma-ray emission below $100 \mathrm{TeV}$, as they require relatively long integration times of up to a year to reach the required sensitivity, whereas the gamma-ray flux is declining on shorter time scales. However, their all-sky monitoring capabilities could lead to early detection of unexpectedly bright gamma-ray SNe events.

\section{Conclusion}

We selected a sample of nine type II SNe that were observed by chance with H.E.S.S. within one year after the SN event, and in addition we triggered ToO observations on SN 2016adj. No significant gamma-ray signal has been detected from any of these ten $\mathrm{SNe}$ and we derived flux upper limits of the order of $10^{-13} \mathrm{TeV} \mathrm{cm}^{-2} \mathrm{~s}^{-1}$.

This result is amending previous efforts (Lennarz \& the H.E.S.S. Collaboration 2013) and complements other recent nondetections, namely the Fermi-LAT studies of type IIn SNe at $\mathrm{GeV}$ energies (Ackermann et al. 2015), the upper limit at $\mathrm{TeV}$ energies established by the MAGIC collaboration for the closest type Ia SN 2014J (Ahnen et al. 2017), and a recent search for $\mathrm{GeV}$ emission from super luminous SNe using Fermi-LAT data by Renault-Tinacci et al. (2018). Concerning SN 2016adj, the H.E.S.S. UL is the first constraint derived on this nearby SN event in the gamma-ray domain. The lack of gamma-ray detection reported here, however, does not necessarily indicate that the early phase of SN evolution is not generally conducive to $\mathrm{CR}$ acceleration. Instead, the non-detection suggests that it does not occur in this subset of the SNe, which have CSM that are not likely to be dense enough for particle acceleration.

Using the model developed in Dwarkadas (2013), we expressed our ULs in terms of constraints on the mass-loss rates of the SN progenitors, which turn out to be a few times higher than, or of the same order as, the estimated mass-loss rate for the close-by radio-bright SN 1993J. With the same model, we predicted that objects with a mass-loss rate of the order of $10^{-4} M_{\odot} \mathrm{yr}^{-1}$ and distance of $\sim 10 \mathrm{Mpc}$ could be detected very early after the outburst by the current generation of telescopes and a fortiori by the next generation, namely the Cherenkov Telescope Array, CTA (Acharya et al. 2013). In our study, we did not observe candidates with the required properties for the detection of gamma rays with H.E.S.S., but our model-dependent investigation suggests that core-collapse $\mathrm{SNe}$ will be detected by Cherenkov arrays in the future.

Acknowledgements. The authors would like to thank R. Margutti for her useful input as well as the anonymous referee for his/her constructive comments. R. Simoni thanks M. Renzo and M. Zapartas for helpful discussions. The support of the Namibian authorities and of the University of Namibia in facilitating the construction and operation of H.E.S.S. is gratefully acknowledged, as is the support by the German Ministry for Education and Research (BMBF), the Max Planck Society, the German Research Foundation (DFG), the Helmholtz Association, the Alexander von Humboldt Foundation, the French Ministry of Higher Education, Research and Innovation, the Centre National de la Recherche Scientifique (CNRS/IN2P3 and CNRS/INSU), the Commissariat àl'énergie atomique et aux énergies alternatives (CEA), the U.K. Science and Technology Facilities Council (STFC), the Knut and Alice Wallenberg Foundation, the National Science Centre, Poland grant no.2016/22/M/ST9/00382, the South African Department of Science and Technology and National Research Foundation, the University of Namibia, the National Commission on Research, Science \& Technology of Namibia (NCRST), the Austrian Federal Ministry of Education, Science and
Research and the Austrian Science Fund (FWF), the Australian Research Council (ARC), the Japan Society for the Promotion of Science and by the University of Amsterdam. We appreciate the excellent work of the technical support staff in Berlin, Zeuthen, Heidelberg, Palaiseau, Paris, Saclay, Tübingen, and in Namibia in the construction and operation of the equipment. This work benefitted from services provided by the H.E.S.S. Virtual Organisation, supported by the national resource providers of the EGI Federation.

\section{References}

Abeysekara, A. U., Alfaro, R., Alvarez, C., et al. 2013, Astropart. Phys., 50, 26 Acharya, B., Actis, M., Aghajani, T., et al. 2013, Astropart. Phys., 43, 3 Ackermann, M., Ajello, M., Allafort, A., et al. 2013, Science, 339, 807 Ackermann, M., Arcavi, I., Baldini, L., et al. 2015, ApJ, 807, 169

Aharonian, F., Akhperjanian, A. G., Bazer-Bachi, A. R., et al. 2006, A\&A, 457, 899

Aharonian, F., Akhperjanian, A. G., Anton, G., et al. 2009, ApJ, 695, L40

Ahnen, M. L., Ansoldi, S., Antonelli, L. A., et al. 2017, A\&A, 602, A98

Arcavi, I., Hosseinzadeh, G., Brown, P. J., et al. 2017, ApJ, 837, L2

Bartel, N., Bietenholz, M. F., Rupen, M. P., et al. 2002, ApJ, 581, 404 Bell, A. R. 2004, MNRAS, 353, 550

Bell, A., Schure, K., Reville, B., \& Giacinti, G. 2013, MNRAS, 431, 415

Berge, D., Funk, S., \& Hinton, J. 2007, A\&A, 466, 1219

Cardillo, M., Amato, E., \& Blasi, P. 2015, Astropart. Phys., 69, 1

Chakraborti, S., Ray, A., Smith, R., et al. 2013, ApJ, 774, 30

Chevalier, R. A. 1982a, ApJ, 258, 790

Chevalier, R. A. 1982b, ApJ, 259, 302

Chevalier, R. A., \& Fransson, C. 1994, ApJ, 420, 268

Chevalier, R. A., \& Fransson, C. 2017, Handbook of Supernovae (AG, Switzerland: Springer International Publishing), 875

Chevalier, R. A., \& Soderberg, A. M. 2010, ApJ, 711, L40

Das, S., \& Ray, A. 2017, ApJ, 851, 138

Davies, B., \& Beasor, E. 2018, MNRAS, 474, 2116

de Jager, C., Nieuwenhuijzen, H., \& van der Hucht, K. A. 1988, A\&AS, 72, 259

de Naurois, M. 2012, PhD thesis, Université Pierre et Marie Curie - Paris VI

de Naurois, M., \& Rolland, L. 2009, Astropart. Phys., 32, 231

Drury, L. O., Aharonian, F. A., \& Voelk, H. J. 1994, A\&A, 287, 959

Dwarkadas, V. V. 2013, MNRAS, 434, 3368

Fransson, C., \& Björnsson, C.-I. 1998, ApJ, 509, 861

Fuller, J. 2017, MNRAS, 470, 1642

Gandhi, P., Yamanaka, M., Tanaka, M., et al. 2013, ApJ, 767, 166

Ginzburg, V. L., \& Syrovatskii, S. I. 1964, The Origin of Cosmic Rays (Oxford: Pergamon Press)

Giuliani, A., Cardillo, M., Tavani, M., et al. 2011, ApJ, 742, L30

Gutiérrez, C. P., Anderson, J. P., Hamuy, M., et al. 2017, ApJ, 850, 90

Harris, G. L. H. 2010, PASA, 27, 475

Helder, E. A., Vink, J., Bykov, A. M., et al. 2012, Space Sci. Rev., 173, 369

Hewitt, J. W., \& Lemoine-Goumard, M. 2015, C. R. Phys., 16, 674

Horiuchi, S., Beacom, J. F., Kochanek, C. S., et al. 2011, ApJ, 738, 154

Katz, B., Sapir, N., \& Waxman, E. 2011, Proc. IAU, 7, 274

Lennarz, D., \& the H.E.S.S. Collaboration 2013, ArXiv e-prints [arXiv: 1307.7727$]$

Li, T.-P., \& Ma, Y.-Q. 1983, ApJ, 272, 317

Li, W., Chornock, R., Leaman, J., et al. 2011, MNRAS, 412, 1473

Marcowith, A., Renaud, M., Dwarkadas, V., \& Tatischeff, V. 2014, Nucl. Phys. B Proc. Suppl., 256, 94

Marcowith, A., Dwarkadas, V. V., Renaud, M., Tatischeff, V., \& Giacinti, G. 2018, MNRAS, 479, 4470

Marples, P., Bock, G., \& Parker, S. 2016, ATel, 8651

Mattila, S., Fraser, M., Smartt, S. J., et al. 2013, MNRAS, 431, 2050

Mauron, N., \& Josselin, E. 2011, A\&A, 526, A156

Moriya, T., Tominaga, N., Blinnikov, S. I., Baklanov, P. V., \& Sorokina, E. I. 2011, MNRAS, 415, 199

Murase, K., Thompson, T. A., Lacki, B. C., \& Beacom, J. F. 2011, Phys. Rev. D, 84, 043003

Murase, K., Thompson, T. A., \& Ofek, E. O. 2014, MNRAS, 440, 2528

Ofek, E. O., Sullivan, M., Shaviv, N. J., et al. 2014, ApJ, 789, 104

O'Sullivan, E., Ponman, T. J., Kolokythas, K., et al. 2017, MNRAS, 472, 1482

Ouchi, R., \& Maeda, K. 2017, ApJ, 840, 90

Parsons, R. D., \& Hinton, J. A. 2014, Astropart. Phys., 56, 26

Pooley, D., Lewin, W. H. G., Fox, D. W., et al. 2002, ApJ, 572, 932

Renault-Tinacci, N., Kotera, K., Neronov, A., \& Ando, S. 2018, A\&A, 611, A45

Smartt, S. J., Maund, J. R., Hendry, M. A., et al. 2004, Science, 303, 499 
Smartt, S. J., Eldridge, J. J., Crockett, R. M., \& Maund, J. R. 2009, MNRAS, 395,1409

Smith, N. 2014, ARA\&A, 52, 487

Smith, N., Chornock, R., Li, W., et al. 2008, ApJ, 686, 467

Smith, N., Hinkle, K. H., \& Ryde, N. 2009, AJ, 137, 3558

Smith, N., Li, W., Filippenko, A. V., \& Chornock, R. 2011, MNRAS, 412, 1522

Stritzinger, M., Hsiao, E. Y., Morrell, N., et al. 2016, ATel, 8657

Strong, A. W., Moskalenko, I. V., Reimer, O., Digel, S., \& Diehl, R. 2004, A\&A, 422, L47

Strong, A. W., Moskalenko, I. V., \& Ptuskin, V. S. 2007, Ann. Rev. Nucl. Part. Sci., 57, 285

Sugerman, B., \& Lawrence, S. 2016, ATel, 8759

Tatischeff, V. 2009, A\&A, 499, 191

Thomas, A., Tucker, B. E., Childress, M., et al. 2016, ATel, 8664

Van Dyk, S. D. 2013, AJ, 146, 24

Van Dyk, S. D., Ascenso, J., Wu, Y.-L., et al. 2016, ATel, 8693

Vernetto, S., \& LHAASO, C. 2016, J. Phys. Conf. Ser., 718, 052043

Vink, J. 2012, A\&ARv, 20, 49

Voelk, H. J., \& Biermann, P. L. 1988, ApJ, 333, L65

Völk, H. J., Berezhko, E. G., \& Ksenofontov, L. T. 2005, A\&A, 433, 229

Weiler, K. 2006, KITP Conference: Supernova and Gamma-Ray Burst Remnants

Zirakashvili, V. N., \& Ptuskin, V. S. 2016, Astropart. Phys., 78, 28

${ }^{1}$ Centre for Space Research, North-West University, Potchefstroom 2520, South Africa

2 Universität Hamburg, Institut für Experimentalphysik, Luruper Chaussee 149, 22761 Hamburg, Germany

${ }^{3}$ Max-Planck-Institut für Kernphysik, PO Box 103980, 69029 Heidelberg, Germany

${ }^{4}$ Dublin Institute for Advanced Studies, 31 Fitzwilliam Place, Dublin 2, Ireland

${ }^{5}$ High Energy Astrophysics Laboratory, RAU, 123 Hovsep Emin St Yerevan 0051, Armenia

6 Yerevan Physics Institute, 2 Alikhanian Brothers St., 375036 Yerevan, Armenia

7 Institut für Physik, Humboldt-Universität zu Berlin, Newtonstr. 15, 12489 Berlin, Germany

${ }^{8}$ Department of Physics, University of Namibia, Private Bag 13301, Windhoek, 12010, Namibia

9 GRAPPA, Anton Pannekoek Institute for Astronomy, University of Amsterdam, Science Park 904, 1098 XH Amsterdam, The Netherlands

${ }^{10}$ Department of Physics and Electrical Engineering, Linnaeus University, 35195 Växjö, Sweden

11 Institut für Theoretische Physik, Lehrstuhl IV: Weltraum und Astrophysik, Ruhr-Universität Bochum, 44780 Bochum, Germany

12 Institut für Astro- und Teilchenphysik, Leopold-FranzensUniversität Innsbruck, 6020 Innsbruck, Austria

13 School of Physical Sciences, University of Adelaide, Adelaide 5005, Australia

${ }^{14}$ LUTH, Observatoire de Paris, PSL Research University, CNRS, Université Paris Diderot, 5 Place Jules Janssen, 92190 Meudon, France

15 Sorbonne Université, Université Paris Diderot, Sorbonne Paris Cité, CNRS/IN2P3, Laboratoire de Physique Nucléaire et de Hautes Energies, LPNHE, 4 Place Jussieu, 75252 Paris, France

16 Laboratoire Univers et Particules de Montpellier, Université Montpellier, CNRS/IN2P3, CC 72, Place Eugène Bataillon, 34095 Montpellier Cedex 5, France
${ }^{17}$ IRFU, CEA, Université Paris-Saclay, 91191 Gif-sur-Yvette, France

18 Astronomical Observatory, The University of Warsaw, Al. Ujazdowskie 4, 00-478 Warsaw, Poland

19 Aix Marseille Université, CNRS/IN2P3, CPPM, Marseille, France

20 Instytut Fizyki Jạdrowej PAN, ul. Radzikowskiego 152, 31-342 Kraków, Poland

${ }^{21}$ School of Physics, University of the Witwatersrand, 1 Jan Smuts Avenue, Braamfontein, Johannesburg 2050, South Africa

22 Laboratoire d'Annecy de Physique des Particules, Université Grenoble Alpes, Université Savoie Mont Blanc, CNRS, LAPP, 74000 Annecy, France

${ }^{23}$ Landessternwarte, Universität Heidelberg, Königstuhl, 69117 Heidelberg, Germany

${ }^{24}$ Université Bordeaux, CNRS/IN2P3, Centre d'Études Nucléaires de Bordeaux Gradignan, 33175 Gradignan, France

25 Institut für Astronomie und Astrophysik, Universität Tübingen, Sand 1, 72076 Tübingen, Germany

${ }^{26}$ Laboratoire Leprince-Ringuet, École Polytechnique, UMR 7638, CNRS/IN2P3, Institut Polytechnique de Paris, 91128 Palaiseau, France

27 APC, AstroParticule et Cosmologie, Université Paris Diderot, CNRS/IN2P3, CEA/Irfu, Observatoire de Paris, Sorbonne Paris Cité, 10, rue Alice Domon et Léonie Duquet, 75205 Paris Cedex 13, France

${ }^{28}$ Université Grenoble Alpes, CNRS, IPAG, 38000 Grenoble, France

29 Department of Physics and Astronomy, The University of Leicester University Road, Leicester, LE1 7RH, UK

30 Nicolaus Copernicus Astronomical Center, Polish Academy of Sciences, ul. Bartycka 18, 00-716 Warsaw, Poland

31 Institut für Physik und Astronomie, Universität Potsdam, KarlLiebknecht-Strasse 24/25, 14476 Potsdam, Germany

32 Friedrich-Alexander-Universität Erlangen-Nürnberg, Erlangen Centre for Astroparticle Physics, Erwin-Rommel-Str. 1, 91058 Erlangen, Germany

${ }^{33}$ DESY, 15738 Zeuthen, Germany

34 Obserwatorium Astronomiczne, Uniwersytet Jagielloński, ul. Orla 171, 30-244 Kraków, Poland

35 Centre for Astronomy, Faculty of Physics, Astronomy and Informatics, Nicolaus Copernicus University, Grudziadzka 5, 87-100 Torun, Poland

36 Department of Physics, University of the Free State, PO Box 339, Bloemfontein 9300, South Africa

37 Department of Physics, Rikkyo University, 3-34-1 Nishi-Ikebukuro, Toshima-ku, Tokyo 171-8501, Japan

${ }^{38}$ Kavli Institute for the Physics and Mathematics of the Universe (WPI), The University of Tokyo Institutes for Advanced Study (UTIAS), The University of Tokyo, 5-1-5 Kashiwa-no-Ha, Kashiwa City, Chiba 277-8583, Japan

39 Department of Physics, The University of Tokyo, 7-3-1 Hongo, Bunkyo-ku, Tokyo 113-0033, Japan

40 RIKEN, 2-1 Hirosawa, Wako, Saitama 351-0198, Japan

${ }^{41}$ Now at Physik Institut, Universität Zürich, Winterthurerstrasse 190, 8057 Zürich, Switzerland

42 Now at Institut de Ciències del Cosmos (ICC UB), Universitat de Barcelona (IEEC-UB), Martí Franquès 1, 08028 Barcelona, Spain

43 School of Sciences, University of New South Wales, Australian Defence Force Academy, Canberra, ACT 2600, Australia 


\section{Appendix A: Statistical analysis}

Table A.1. Observed statistics for each SN (see text).

\begin{tabular}{llccccccc}
\hline \hline SNe & $N_{\text {on }}$ & $N_{\text {off }}$ & $\alpha$ & $N_{\text {excess }}$ & Sig & $\begin{array}{c}\text { Livetime } \\
\text { (h) }\end{array}$ & $\begin{array}{c}\text { Obs. coverage } \\
\text { (days) }\end{array}$ & $\begin{array}{c}\text { Average time } \\
\text { (days) }\end{array}$ \\
\hline SN 2004cx & 169 & 10387 & 0.015 & 8.7 & 0.7 & 40 & -6 to 359 & 180 \\
SN 2005dn & 571 & 11452 & 0.053 & -39 & -1.5 & 53 & -3 to 364 & 120 \\
SN 2008bk & 50 & 3652 & 0.018 & -18 & -2.3 & 9.6 & $98-211$ & 136 \\
SN 2008bp & 32 & 1860 & 0.017 & 1.1 & 0.2 & 4.7 & $272-282$ & 282 \\
SN 2008ho & 9 & 369 & 0.030 & -2.3 & -0.7 & 1.4 & $34-34$ & 34 \\
SN 2009hf & 43 & 1404 & 0.029 & 3.3 & 0.5 & 4.0 & $124-134$ & 133 \\
SN 2009js & 14 & 711 & 0.015 & 3.4 & 1 & 4.8 & $1-35$ & 17.5 \\
SN 2011ja & 37 & 620 & 0.053 & 4.51 & 0.75 & 3.4 & $91-131$ & 111 \\
SN 2012cc & 7 & 660 & 0.013 & -1.9 & -0.7 & 3.0 & $53-343$ & 255 \\
SN 2016adj & 624 & 8573 & 0.070 & 22 & 0.9 & 13 & $3-10$ & 7 \\
\hline
\end{tabular}

Notes. Sig stands for significance and the observation range gives the number of days since the SN discovery date for the first - last observation run. The average time is the exposure-weighted mean time in days since the discovery date.

\section{Appendix B: Lightcurves}
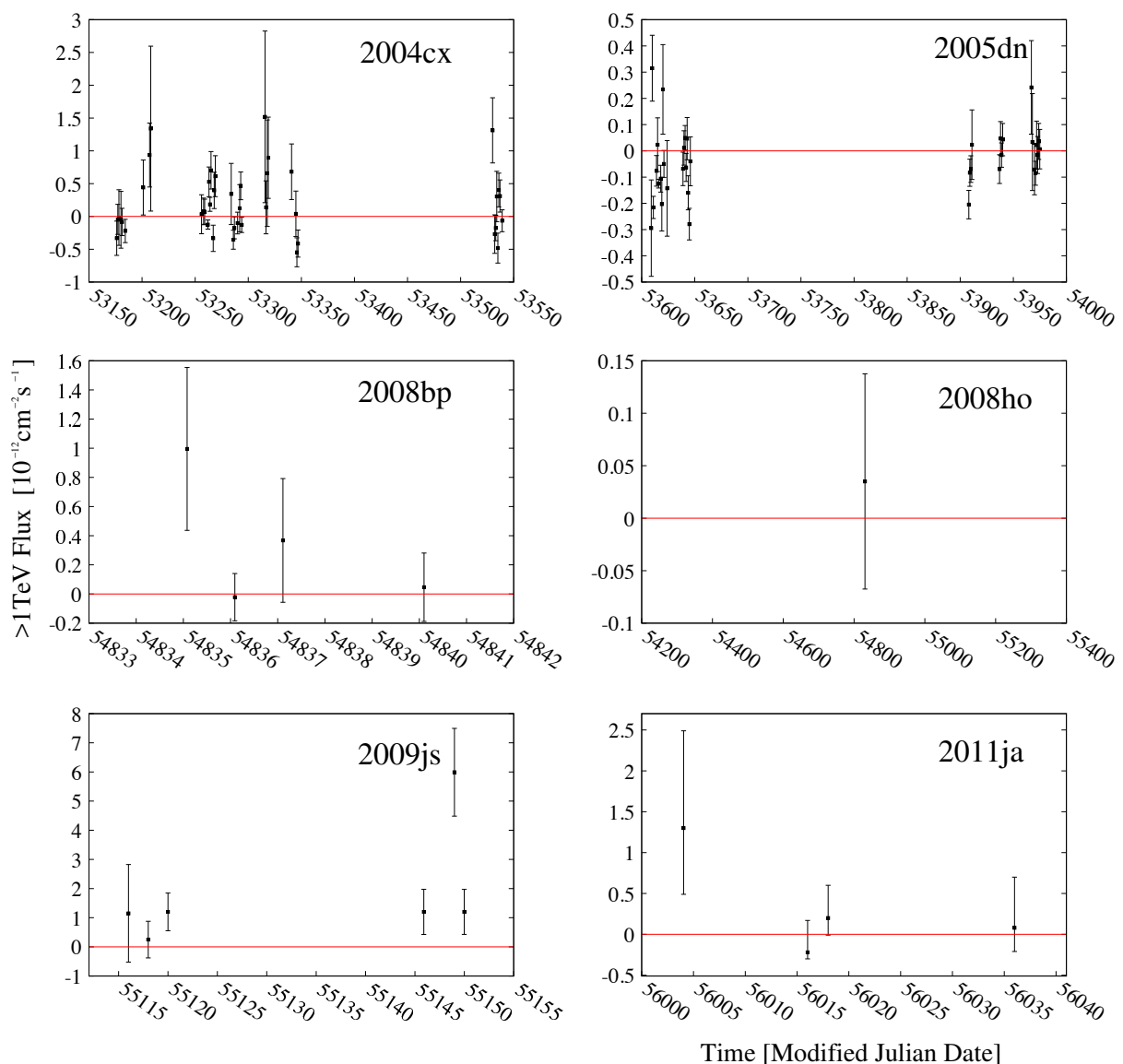
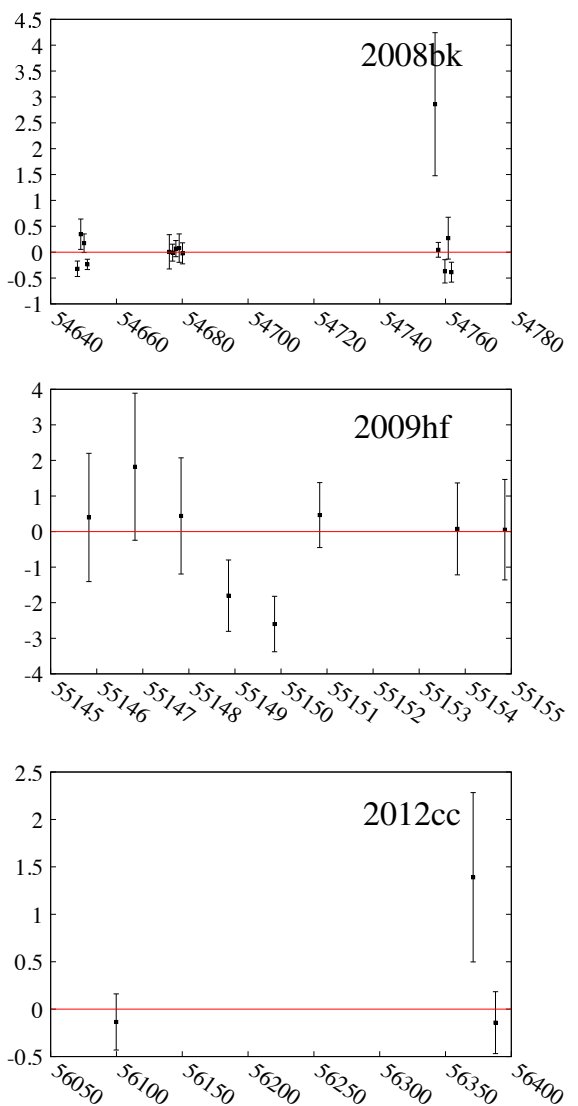

Fig. B.1. Time evolution binned on a nightly basis of the integrated flux above $1 \mathrm{TeV}$ as measured with H.E.S.S. towards the nine serendipitously observed SNe considered in this study. The red line outlines the zero level. 